\title{
COUPLING AMONG IDENTIFIED CELLS IN MAMMALIAN NERVOUS SYSTEM CULTURES ${ }^{1}$
}

\author{
H. KETTENMANN, R. K. ORKAND, ${ }^{2}$ AND MELITTA SCHACHNER ${ }^{3}$ \\ Department of Neurobiology, University of Heidelberg, Im Neuenheimer Feld 504, D-6900 \\ Heidelberg, Federal Republic of Germany
}

Received June 21, 1982; Revised September 8, 1982; Accepted September 9, 1982

\begin{abstract}
Cell coupling can be demonstrated amoung groups of oligodendrocytes, astrocytes, and fibroblasts or fibroblast-like cells, but not for dorsal root ganglion neurons, Schwann cells, or macrophages in mouse or rat cell cultures. Neurons were identified by their ability to generate action potentials. Non-neuronal cells were recognized immunologically by their ability to react with a varicty of rhodamine-labeled cell type-specific antibodies. Intracellular injection of the gap junction permeable fluorescent dye Lucifer Yellow and ionic current were used to establish the presence of coupling among these identified cells. Coupling under the culture conditions used in this study does not represent a random membrane interaction between closely apposed cells but rather a form of communication among restricted populations.
\end{abstract}

Cell coupling permits the cytoplasmic exchange of ions and small molecules among cells. It is widespread in living systems (Gilula and Epstein, 1976; Bennett et al., 1981). This exchange occurs across intercellular aqueous pathways at specialized cell-to-cell contacts termed gap junctions (Furshpan and Potter, 1968; Michalke and Loewenstein, 1971). In the nervous system such junctions have functional implications beyond those involving metabolic cooperation. In neurons these low resistance cell connections permit the rapid propagation of electrical impulses via electrical synapses (Bennett, 1977). For glial cells these junctions are the presumed site of ionic transfer involved in the spatial buffering of potassium (Orkand et al., 1966; Gardner-Medwin et al., 1981).

In mammalian nervous system cultures, coupling among neurons has rarely been observed (Ransom et al., 1977a). There is one report of electrical coupling between cells presumed to be astrocytes (Moonen and Nelson, 1978). Although neurons may readily be identified in nervous system cultures by their impulse activity, the

\footnotetext{
${ }^{1}$ This work was supported by Deutsche Forschungsgemeinschaft (Scha 185/6), Studienstiftung des deutschen Volkes (H. K.), Deutscher Akademischer Auslandsdienst and United States Public Health Service Grant NS-12253 (R. K. O.). We wish to thank U. Sonnhof for stimulating discussions and G. Fischer, Jutta Schnitzer, and Ilse Sommer for antibodies and cultures. We are grateful to Drs. A. Bignami, Doris Dahl, Kay Ficlds, and G. Hämmerling for gifts of antibodies.

${ }^{2}$ Present address: Department of Physiology and Pharmacology, University of Pennsylvania, School of Dental Medicine, Philadelphia, PA 19104.

${ }^{3}$ To whom correspondence should be addressed.
}

identification of glial and other non-neuronal elements is not yet possible with electrophysiological methods. Recently, a variety of immunological markers have become available which permit identification of cell types after reaction with fluorochrome-labeled cell type-specific antibodies (for review, see Schachner, 1982).

In the present study we have combined the techniques for electrophysiological determination of low resistance pathways between cells and intracellular injection of the gap junction permeable dye Lucifer Yellow (Stewart, 1981) with immunological identification of cell types. With these techniques we looked for coupling among dorsal root ganglion neurons, Schwann cells, astrocytes, oligodendrocytes, fibroblasts and fibroblast-like cells, and macrophages in mammalian nervous system cultures.

\section{Materials and Methods}

Cell cultures. Explant cultures of spinal cord, including dorsal root ganglia (DRG), were prepared from 14-dayold mouse embryos as described previously (Kettenmann et al., 1983). Explants were plated on collagen-coated glass coverslips and maintained in plastic culture dishes at $35.5^{\circ} \mathrm{C}$ and $3.5 \% \mathrm{CO}_{2}$ for 3 to 6 weeks. The culture medium, Eagle's Basal Medium with Earle's salts supplemented with $10 \%$ calf serum, was changed weekly. In some cases, recordings from astrocytes were carried out in cultures of dissociated early postnatal mouse cerebellum cultured in defined medium for 3 weeks under conditions described by Fischer (1982). Schwann cells were obtained from postnatal rat dorsal root ganglia cultured under the same conditions as mouse spinal cord. 
Electrophysiology. Three types of intracellular electrodes were used. Double barreled $\theta$ glass electrodes were used for current passing, potential recording, and dye injection. The barrel for potential recording and current passing was filled with $3 \mathrm{~m}$ potassium acetate (10 to 25 megohms) and that for staining with $4 \%$ Lucifer Yellow (kindly provided by W. Stewart). Single barreled 3 M potassium acetate electrodes ( 20 to 60 megohms) made from dotted glass capillaries were used for either potential recording or current passing. In some experiments, where accurate membrane potential recording was not required, Lucifer Yellow was injected from single barrel electrodes filled with $4 \%$ dye in $0.5 \mathrm{M}$ lithium chloride (50 to 100 megohms). The culture dish was mounted on the stage of a Zeiss inverted microscope and maintained at 30 to $35^{\circ}$ in a $3.5 \% \mathrm{CO}_{2}$ atmosphere ( $\mathrm{pH} 7.2$ to 7.4 ). The reference electrode was a $\mathrm{Ag}-\mathrm{AgCl}$ pellet connected to the bathing fluid via a strip of filter paper (MelittaWerke, Minden, Fed. Rep. Germany). Cells were selected visually at a magnification of $\times 250$ for study. Penetration of pairs of cells was accomplished with the aid of two step-motor driven micromanipulators (Sonnhof et al., 1982). These enabled movements of microelectrodes in steps as small as $125 \mathrm{~nm}$ at high accelerations. For relatively thick cells, like dorsal root ganglion neurons and oligodendrocytes, impalement was accomplished with steps of $1.9 \mu \mathrm{m}$ at a velocity of $5 \mathrm{~mm} / \mathrm{sec}$. Thin cells, such as fibroblasts and astrocytes, could only be successfully impaled with steps of 0.6 to $1 \mu \mathrm{m}$. Occasionally, penetration was aided by current pulses through the microelectrode.

Recording electrodes were connected to two WPI M707 microprobe systems. The signals were displayed on a Nicolet Digital Oscilloscope and a separate chart recorder. A Hewlett-Packard 7004 B X-Y plotter was used to make permanent records of the signals stored in the oscilloscope memory. After electrical recording, cells were injected with Lucifer Yellow by passing a hyperpolarizing current of 5 to $20 \mathrm{nA}$ for 0.5 to $5 \mathrm{~min}$. We attempted to minimize the hyperpolarizing current necessary for dye injection or to test electrical coupling because following large hyperpolarizations, to greater than $120 \mathrm{mV}$, the cells frequently detached from the culture either during withdrawal of the electrode or during the staining and fixation procedures. The inverted microscope was fitted with a 100-watt tungsten-halogen lamp (kindly provided by B. Salzberg), a BG 12 excitor filter, and a 430-nm barrier filter. This enabled assessment of the adequacy of dye injection during the passage of the current. Following injection of one cell of a pair, a diagram of the anatomical relations of the two cells was made to enable identification of both cells following immunological staining. Adequately injected cells were fixed in $4 \%$ paraformaldehyde in phosphate-buffered saline within 0.5 to $200 \mathrm{~min}$.

Immunological methods. Indirect immunofluorescence on cultured cells was carried out as described previously (Schnitzer and Schachner, 1981). Antibodies to 01 antigen were used on live cells to label the cell surface of oligodendrocytes (Sommer and Schachner, 1981). Glial fibrillary acidic (GFA) protein served as a marker for astrocytes, fibronectin for fibroblasts and fibroblast-like cells, mouse histocompatibility antigen $\mathrm{H}$ -
2 for macrophages (Schnitzer and Schachner, 1981), and Ran-1 for Schwann cells (Fields et al., 1978). Rhodamineconjugated anti-immunoglobulin antibodies (rabbit antimouse Ig and goat antirabbit Ig coupled with tetramethylrhodamine, from Dynatech GmbH, Plochingen, Fed. Rep. Germany) served to distinguish labeling of antigens from staining with Lucifer Yellow using a Zeiss fluorescence microscope equipped with epi-illumination and the appropriate filters. For Lucifer Yellow detection, the filter combination consisted of BP 450-490, FT 510, and LP 520; for rhodamine detection, it consisted of BP 545 , FT 580, and LP 590.

\section{Results}

After maintenance of embryonic mouse spinal cord explant cultures for 3 to 6 weeks, an outgrowth zone is observed 2 to $5 \mathrm{~mm}$ from the center of the explant. Within this zone are two distinct types of glial cells, astrocytes and oligodendrocytes. In the outgrowth zone of dorsal root ganglion explants are Schwann cells and DRG neurons. In addition, we find fibroblasts and fibroblast-like cells and macrophages in the periphery of the explant zones. The borders between cell types are not rigid, and often there is considerable overlap due to cell migration. Figure 1 is a generalized diagram of the spatial relations of cells within explant cultures used in this study.

The general plan of the experiments was to penetrate each cell type, look for cell coupling with current and/or dye injection, and then identify the cell types by indirect immunofluorescence.

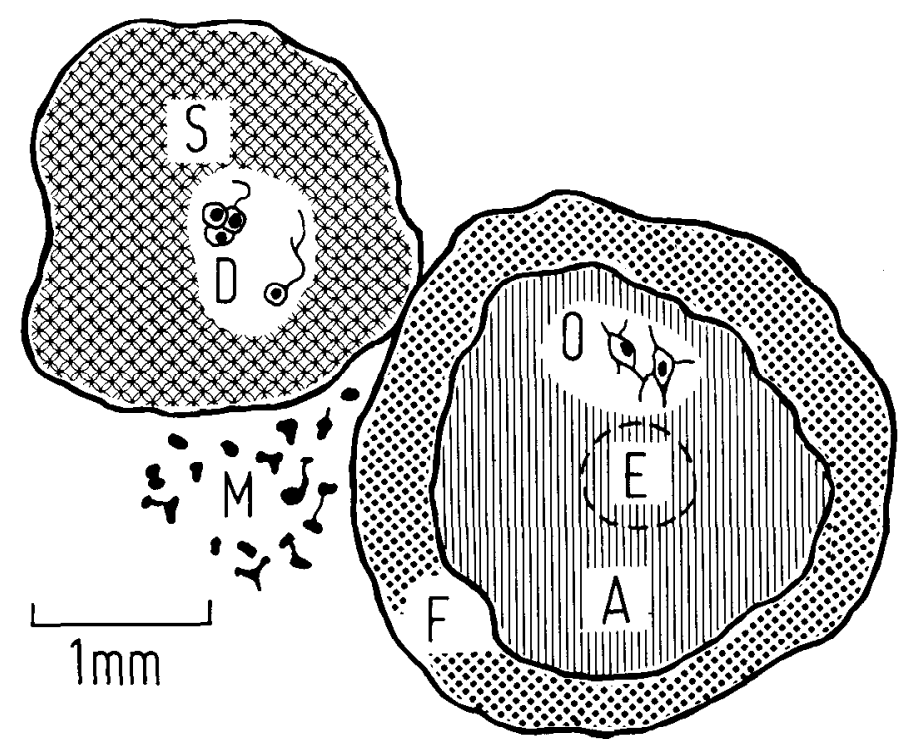

Figure 1. Schematic diagram of topographical relationship between cell types in spinal cord and dorsal root ganglion in culture systems. The spinal cord explant $(E)$ decreases in size and its center undergoes necrosis within 3 weeks. In the outgrowth zone, oligodendrocytes $(O)$ are found on top of a confluent layer of mostly epithelioid astrocytes $(A)$. Surrounding the astrocyte layer fibroblasts and fibroblast-like cells $(F)$ are observed. DRG cells migrate out of the explant within 1 week in culture and form a sheath of Schwann cells $(S)$ with overlying DRG neurons $(D)$. The cell-sparse region between and outside of explant outgrowth zones contains macrophages $(M)$. 

(A)
(B)

${ }_{\mathrm{mV}}^{10}[$

$\begin{array}{ll}50 \mathrm{msec} & 5 \\ & 10 \\ n A\end{array}$
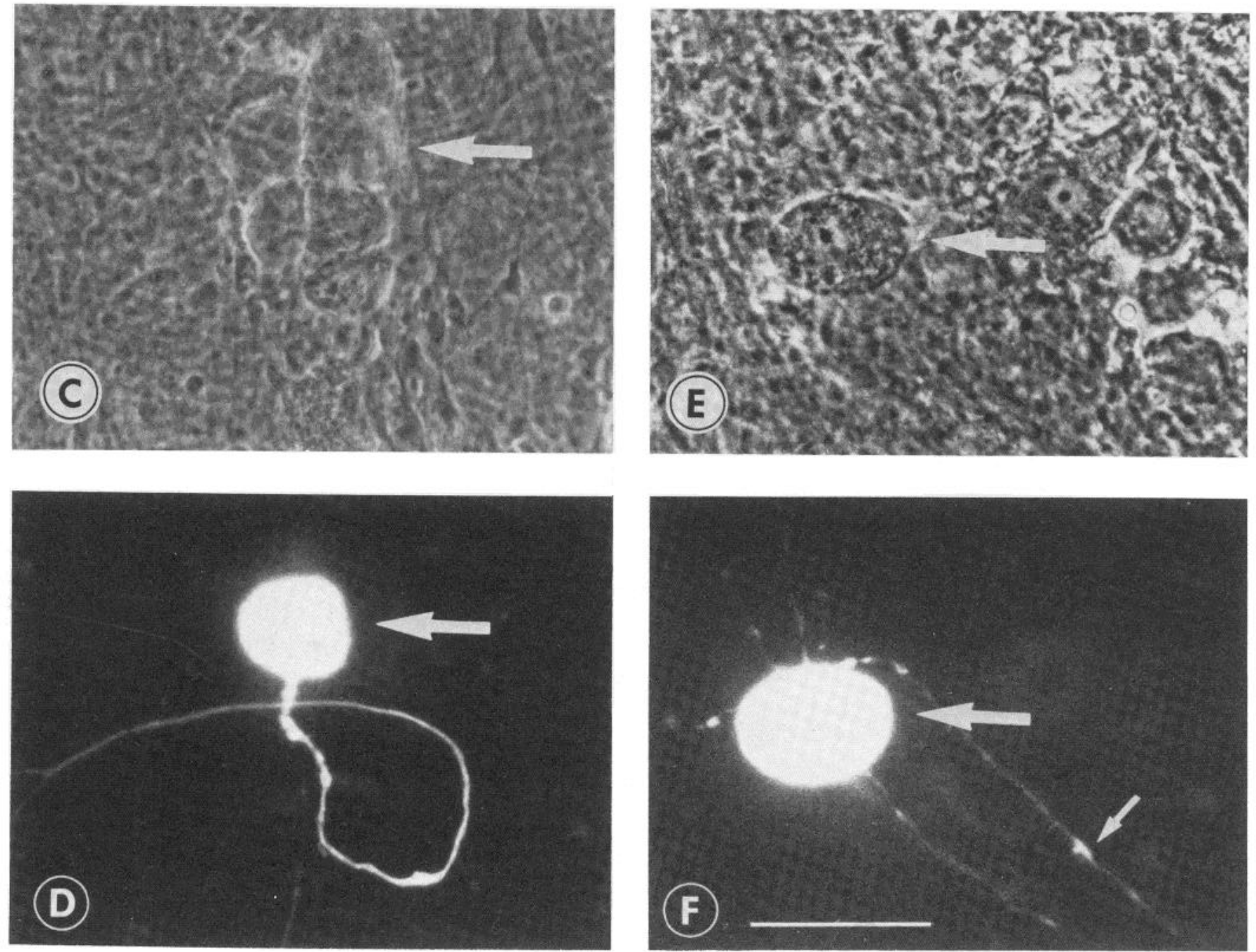

Figure 2. 
Dorsal root ganglia. DRG neurons are lying on top of a sheet of Schwann cells. As shown in Figure 2, they occur either isolated or in groups and were identified by their characteristic morphology (spherical body, 18 to 20 $\mu \mathrm{m}$ in diameter) and ability to generate action potentials (Ransom et al., 1977a).

Sixteen pairs of DRGs were successfully impaled with microelectrodes and recorded from simultaneously. The distance between the cells ranged from 0 (cell bodies appear to touch) to $50 \mu \mathrm{m}$. Neither hyperpolarizing currents up to $25 \mathrm{nA}$ nor a triggered action potential in one cell resulted in a measurable potential change across the membrane of the second cell. The noise level of the recording electrode was generally less than $0.5 \mathrm{mV}$. As the voltage change in the stimulated cell was about 100 $\mathrm{mV}$, this would give an upper level to the coupling ratio of 0.005 (Bennett, 1977).

The Schwann cells of the dorsal root ganglia are closely associated with the neurons and their processes. Recorded membrane potentials were up to about $-50 \mathrm{mV}$ as measured with Li-Lucifer filled electrodes. Nine Schwann cells were injected with Lucifer Yellow in rat cultures and subsequently identified with the rat Schwann cell-specific antibody Ran-1 (Fields et al., 1978). In no case was there spread of dye either to other Schwann cells (Fig. 3) or to the adjacent neurons. Seven cells, with comparable appearance and location in mouse cultures, were injected with the same result. Cells in dorsal root ganglia, although closely apposed, did not exhibit either homologous or heterologous coupling.

Oligodendrocytes. Groups of oligodendrocytes were tentatively identified by their shape and location in the outgrowth zone (Figs. 1 and 3). Following electrical recording and dye injection, they could be conclusively identified by immunological staining with the monoclonal antibody 01 (Sommer and Schachner, 1981; Schachner et al., 1981). Only 16 of 47 pairs of cells with cell bodies separated by less than $100 \mu \mathrm{m}$ were found to be electrically coupled. By contrast, following dye injection into one of the pair and in an additional 25 cells, more than $70 \%$ of the injected oligodendrocytes were found to be dye coupled to at least one and up to as many as five oligodendrocytes. In some cases, the cell body of an oligodendrocyte was neither electrically nor dye coupled to a nearby oligodendrocyte (Fig. 4) but was observed to be dye coupled, via long processes, to a more distant oligodendrocyte. Dye-coupled cells were found as far as $300 \mu \mathrm{m}$ from the injected cell body. Such coupled cells could always be observed to be directly connected to a process of the injected cell (Fig. 5).
The limit for detection of electrical coupling of oligodendrocytes, determined by the amount of current which could be injected and the recording noise level, was the same as that for DRG neurons, coupling ratio of about 0.005 . Assuming an input resistance of 2 megohms (Kettenmann et al., 1983), the coupling ratio for the 16 pairs of coupled oligodendrocytes was $0.1( \pm 0.07 \mathrm{SD}$, range 0.02 to 0.23 )

To test for electrical coupling, cell pairs were selected in which the recorded resting potential was above -50 $\mathrm{mV}$ in each cell. However, even if the resting potential fell markedly (to -10 or $-20 \mathrm{mV}$ ) in one or both cells, the amplitude of the electrotonic potential decreased, but coupled cells did not uncouple. We have no evidence that coupling was strongly dependent either on membrane potential or trans-gap potential.

Astrocytes. In our cultures penetrations of epithelioid astrocytes resulted in a lower proportion of stable membrane potential recordings (greater than $-50 \mathrm{mV}$ ) than from oligodendrocytes. Nevertheless, the results with astrocytes were more consistent in that there was always extensive intercellular coupling among these cells. Cultures containing astrocytes were derived from two sources, spinal cord explants and a cerebellar culture system consisting of astrocytes and neurons (Fischer, 1982). On four occasions we succeeded in recording large membrane potentials from pairs of astrocytes, and all were electrically coupled. Since we did not obtain a measure of the input resistance of single cells, it was not possible to calculate the coupling ratios. Dye injections made into seven cells clearly showed that all were coupled to adjacent cells which in turn spread dye to more distant cells. Figure 6 illustrates electrical and dye coupling in identified astrocytes. Dye and current recordings show that coupled cells usually consisted of a glial fibrillary acidic protein-positive population. In the particular cerebellar culture system occasionally studied vimentinpositive and fibronectin-negative astrocytes which did not express GFA were found coupled to GFA-positive astrocytes. The identification of the astrocytes was made after fixation with paraformaldehyde and ethanol using the astrocyte-specific antibody to GFA protein (Eng et al., 1971; Bignami et al., 1972; Schachner et al., 1977).

Fibroblasts and macrophages. Fibroblasts and macrophages were frequently found in the explant cultures of spinal cord. While macrophages had a distinct morphology which made a tentative identification easy, on visual inspection we could not distinguish fibroblasts from astrocytes with epithelioid morphology. In five cases, cells injected with Lucifer Yellow subsequently

Figure 2. Lack of coupling in DRG neurons. A, During recording from one DRG neuron a second neuron was impaled (upper trace). The action potential triggered by the impalement (lower trace) did not produce a potential change across the membrane of the first cell. The small deflection of the trace from the first cell is the same as that observed with the electrode in the extracellular fluid; it results from current flow in the external medium. $B$, Simultaneous recording from two DRG neurons. Anode break excitation of one neuron (lower trace) produced by injected current (middle trace) through a bridge circuit does not produce a measurable potential change in a second DRG neuron (upper trace). Note increased gain of recordings in upper traces in both $A$ and $B$. $C, D$ and $E, F$ are groups of identical microscopic fields in phase contrast $(C$ and $E)$ and Lucifer Yellow fluorescence $(D$ and $F$ ). $C$, Phase contrast micrograph of a cluster of six DRG neurons. The neuron indicated by the arrow has been injected with Lucifer Yellow. $D$, Fluorescence micrograph of the same group of neurons. Only the injected neuron and its processes contain Lucifer Yellow. $E$ and $F$, Comparable photographs for two closely apposed neurons. The injected neuron has six dye-filled processes (one indicated by small arrow), but no dye has spread to any other cell. Calibration bar represents 50 $\mu \mathrm{m}$ in $D$ to $F$. 

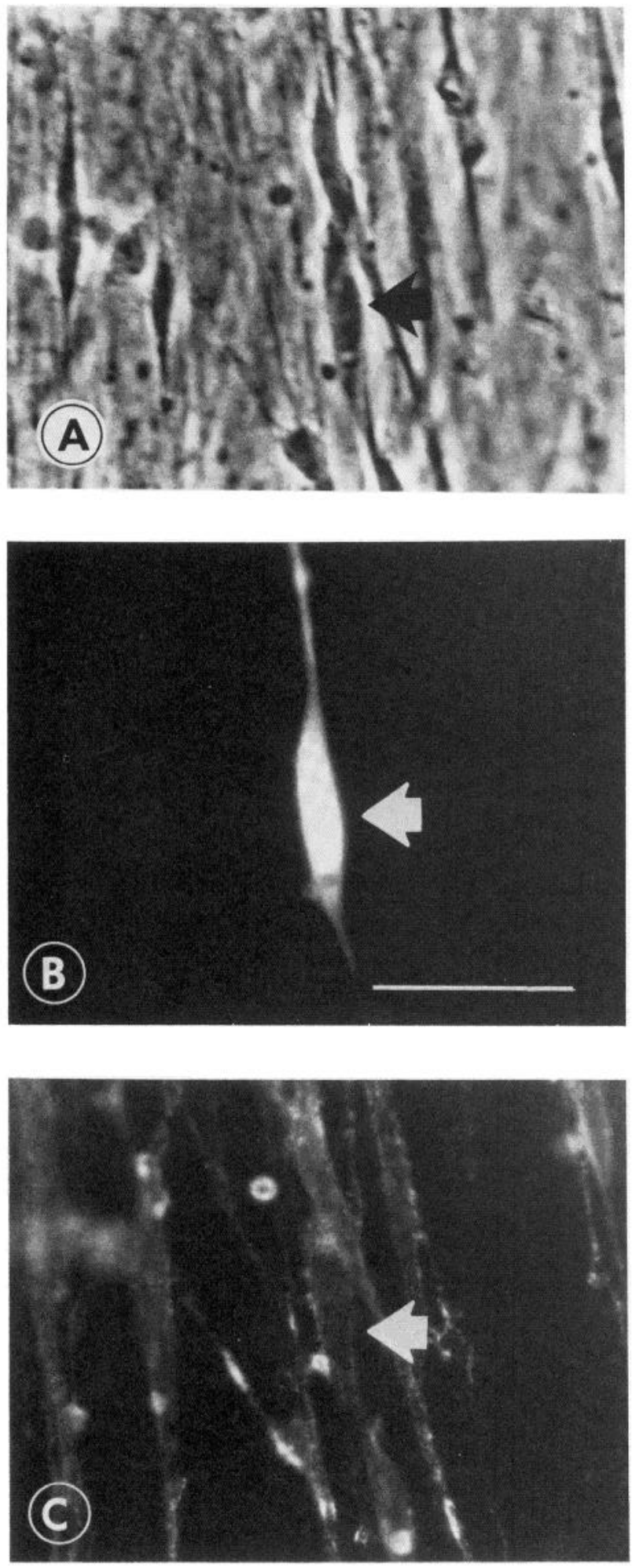

were shown to be fibroblasts or fibroblast-like cells (Fig. 7, $D$ to $F$ ) by immunolabeling for fibronectin (Schachner et al., 1978; Schachner and Willinger, 1979: Schnitzer and Schachner, 1981). In all cases the cells were extensively coupled to other fibroblasts (O'Lague et al., 1970). In 26 cases, macrophages were injected with dye and never found to be coupled (Fig. 7, $A$ to $C$ ). Often macrophages were surrounded by a cell-free zone and no coupling could be expected. However, in several cases macrophages appeared in contact with one another and still there did not appear to be any diffusion of dye between the cells. In four cases, the injected cells were demonstrated to be macrophages by a very intense staining with H-2 antibody (Schnitzer and Schachner, 1981). This strong intensity of immunofluorescence label distinguishes macrophages from astrocytes and fibroblasts, some of which were found to label also for $\mathrm{H}-2$ antigen in cerebellar cultures, but with a markedly lower staining intensity (Schnitzer and Schachner, 1981).

Coupling among different cell types. Based on their anatomical proximity in culture (Fig. 1), a variety of cell coupling combinations are possible among different types of cells. For example, coupling could occur among oligodendrocytes, astrocytes, and fibroblasts or between DRG neurons and Schwann cells. On 10 occasions we recorded simultaneously from identified oligodendrocytes and underlying astrocytes or fibroblasts. In no case was electrical coupling observed. Following 72 successful injections of Lucifer Yellow into oligodendrocytes, detectable dye coupling was observed in the underlying sheath-like cells on only two occasions. As we could not monitor the membrane potential of the cell being injected during the injection, we cannot rule out the possibility that the electrode moved into the underlying cell during the passage of current. The phenomenon was sufficiently rare that it was not feasible to study it further. Neurons growing on top of the astrocytic cell layer were not found to be dye coupled to the astrocytes.

Additional instances of dye coupling between different cell types may have occurred without being detected.

Figure 3. Schwann cells from rat dorsal root ganglion. After injection of Lucifer Yellow into a Schwann cell, the culture was immunolabeled with rhodamine for the Schwann cell-specific antigen Ran-1. In this and the subsequent figures, the greenishyellow fluorescent dye Lucifer Yellow is detected at different excitation and emission wavelengths than the rhodamine-labeled antibody. $A$, Phase contrast micrograph of a group of Schwann cells representing the same microscopic field as shown in fluorescence images $B$ and $C$. $B$, Fluorescence image of Lucifer Yellow distribution. $C$, Indirect immunofluorescence detection of Ran-1 antigen. Calibration bar represents $50 \mu \mathrm{m}$.

Figure 4. Oligodendrocyte coupling. $A$, Spread of electrical current between two cells. Current $(I)$ injected into one cell before $\left(V_{o}\right)$ and after $\left(V_{i}\right)$ penetration of a second cell $0.1 \mathrm{~mm}$ away. $V_{i}-V_{o}$ shows the potential change across the membrane of the second cell due to current injection into the first cell. The cells are electrically coupled. $B$, Same experimental arrangement as in $A$ for a different pair of oligodendrocytes $0.05 \mathrm{~mm}$ apart. Although the injected current was increased more than twice that in $A$, there was no measurable electrical coupling. $C$ to $H$, Distribution of Lucifer Yellow between immunologically identified oligodendrocytes. $C$ to $E$ and $F$ to $H$ are groups of identical microscopic fields in phase contrast $(C, F)$, fluorescence for Lucifer Yellow detection $(D, G)$, and for rhodamine detection of the oligodendrocyte-specific antigen $01(E, H)$. The injected cells are shown by the arrow. $C$ to $E$ illustrate a clearly dye-coupled pair of oligodendrocytes, whereas dye coupling could not be established for the cells in $F$ to $H$. The fluorescence visible to the left of the injected cell in $G$ represents nonspecific background fluorescence. Calibration bar represents $100 \mu \mathrm{m}$. 
(A)

$\begin{array}{ll}V_{0} & 20 \\ v_{i} & m V\end{array}$

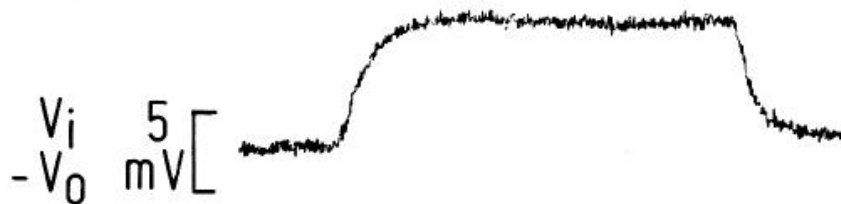

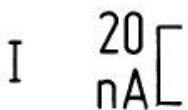
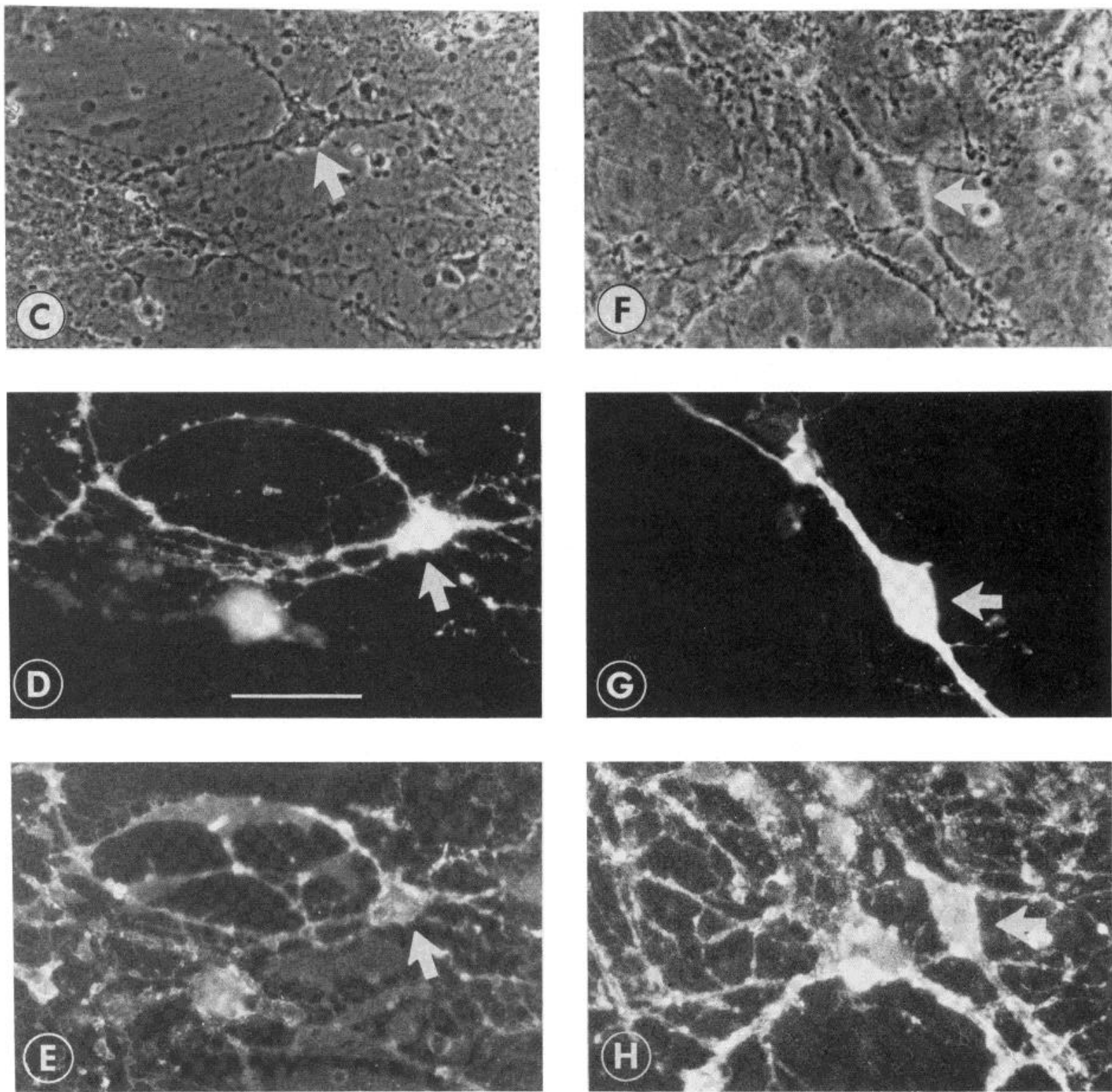

(B)

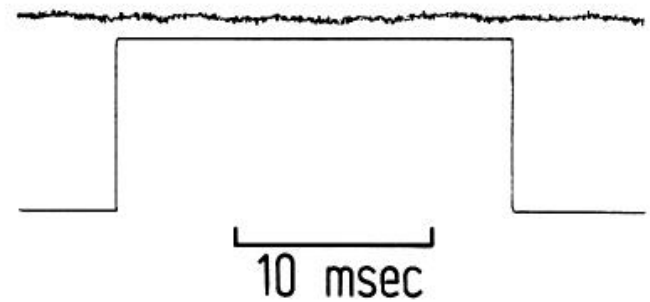

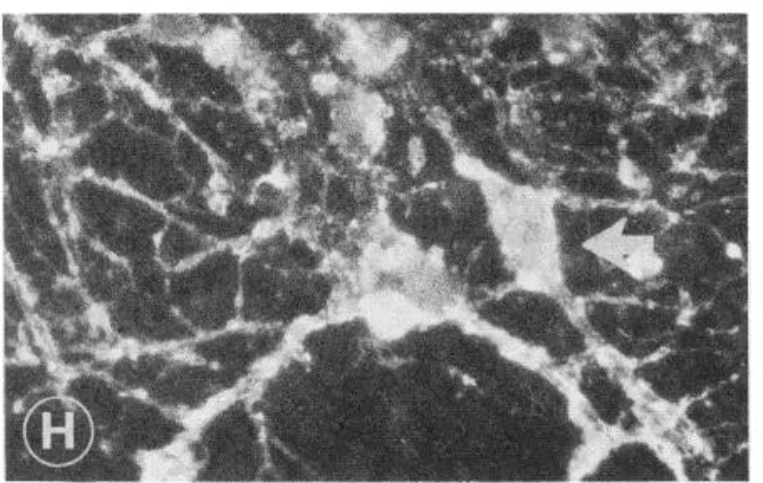

Figure 4. 
Cultures fixed with paraformaldehyde had a low level of fluorescence at the wavelength used for detection of Lucifer Yellow. This background sets the limit for detecting coupling with a low coupling ratio. However, within the limitations of techniques used in the present study, we were unable to demonstrate coupling between different types of cells.
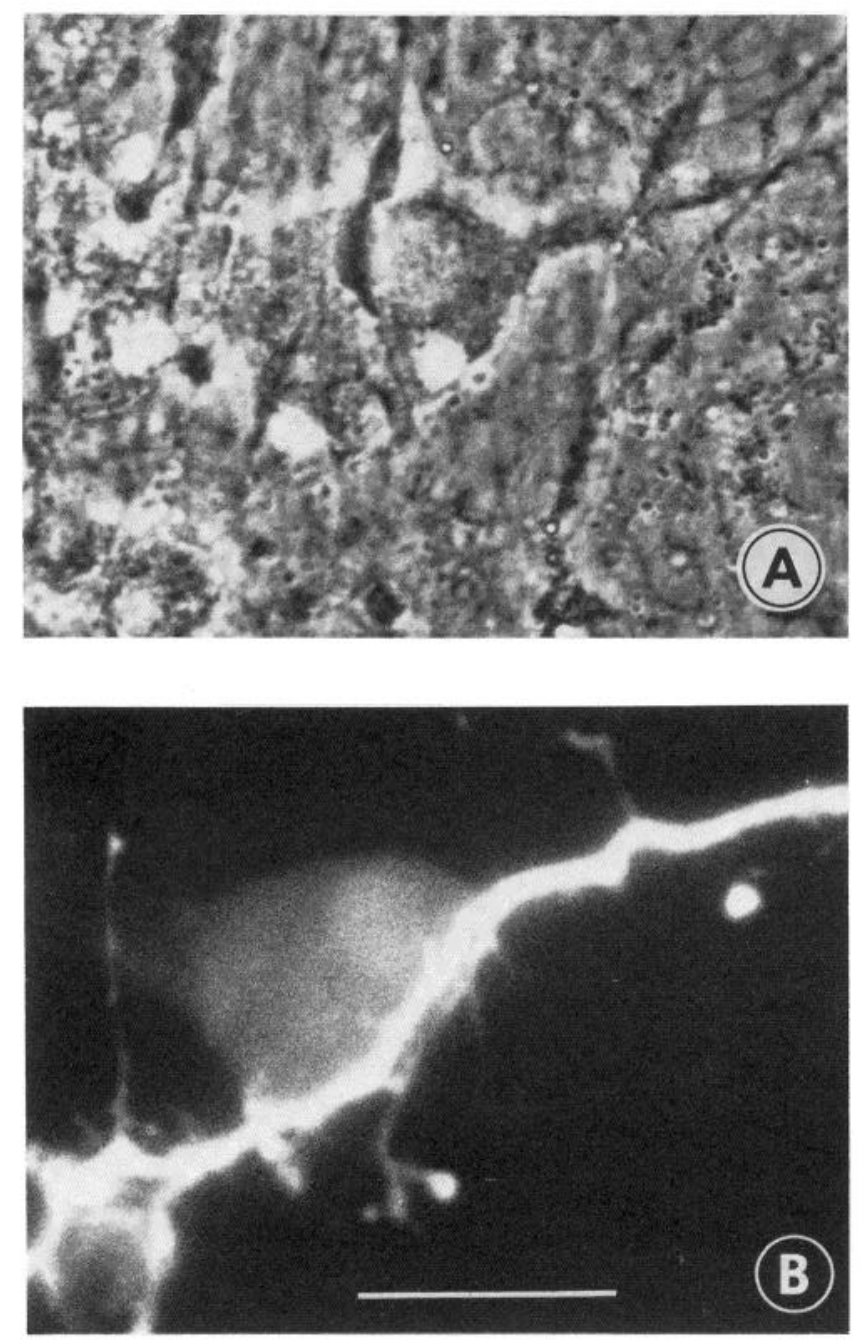

\section{Discussion}

The use of nervous system cell cultures promises to be a powerful tool in studying cellular interactions in the developing and differentiated nervous system. A necessary prerequisite for such studies is the availability of criteria for identification of cell types. Although the
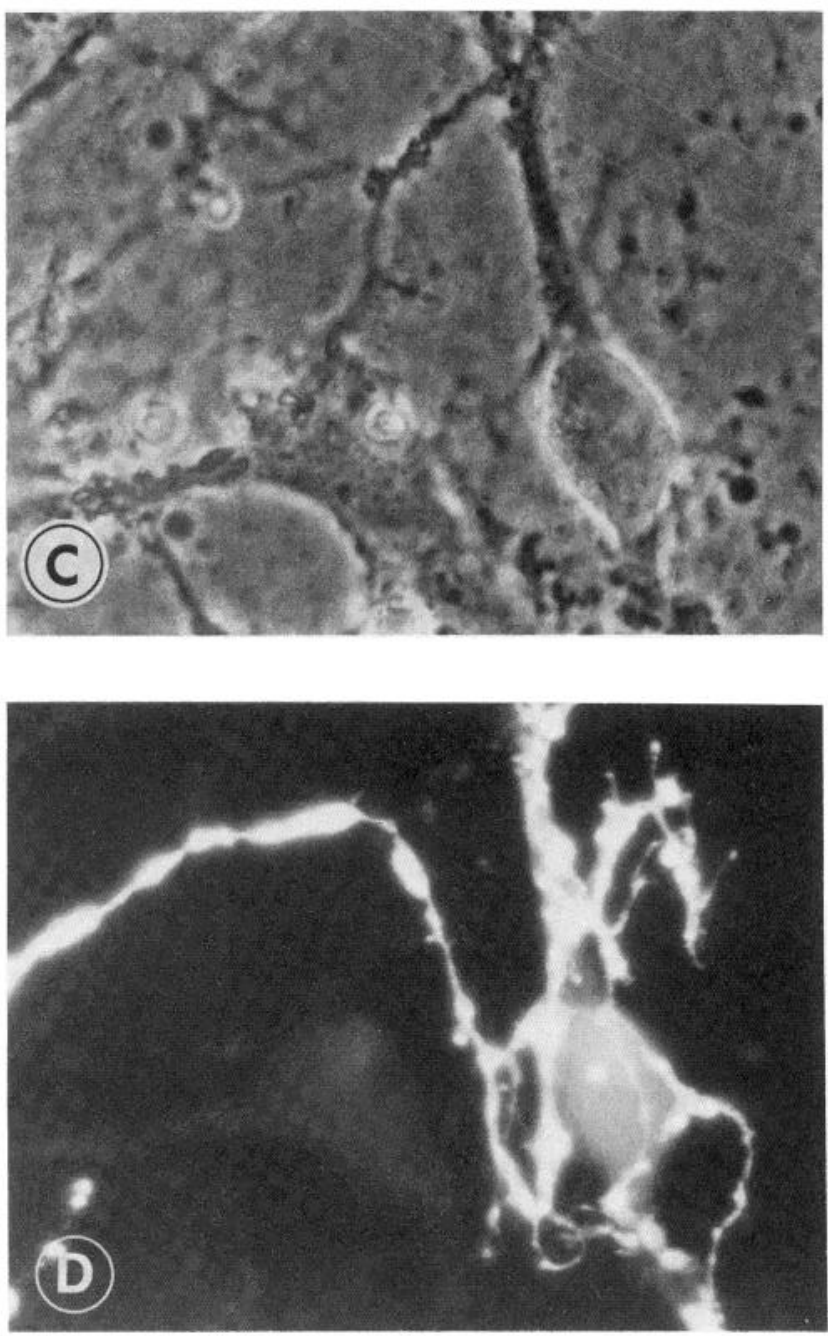

Figure 5. Coupling between distant oligodendrocytes via processes. $A, B$ and $C, D$ are groups of identical microscopic fields. $A$ and $C$ are phase contrast micrographs; $B$ and $D$ are corresponding fluorescence micrographs showing the distribution of Lucifer Yellow. Processes which were found more than $100 \mu \mathrm{m}$ from the Lucifer dye-injected oligodendrocyte cell body are shown as they course over other cell bodies. The dye has readily spread from the process of one cell into the soma of another cell. Calibration bar represents $50 \mu \mathrm{m}$.

Figure 6. Coupling among astrocytes. $A$, Electrical coupling. Current $(I)$ injected in one astrocyte before $\left(V_{o}\right)$ and after $\left(V_{i}\right)$ penetration of a second astrocyte $0.1 \mathrm{~mm}$ away. The cells are electrically coupled. $B$ to $D$ constitute a group of identical microscopic fields showing a dye-coupled group of astrocytes in a cerebellar culture system. $E$ to $G$ comprise a group of identical microscopic fields showing dye-coupled astrocytes from spinal cord. $B, E$ are phase contrast micrographs of fluorescence images $C, D$ and $F, G$, respectively. $C$ and $F$ show the distribution of Lucifer Yellow. $D$ and $G$ show the immunofluorescence distribution of GFA as a marker for astrocytes. The injected cells are indicated by arrows. Note that in the cerebellar culture system one of the coupled cells is not GFA positive, and a second cell is only weakly positive (upper left). Inasmuch as cell types, other than neurons or astroglia, are not present in the culture system (Fischer, 1981), the coupled and GFA-negative or weakly positive cells are immature astrocytes. Also, there is no dye coupling to the overlying small cerebellar neurons which appear as black dots by phase contrast microscopy $(A)$. Calibration bar represents $100 \mu \mathrm{m}$. 
(A)
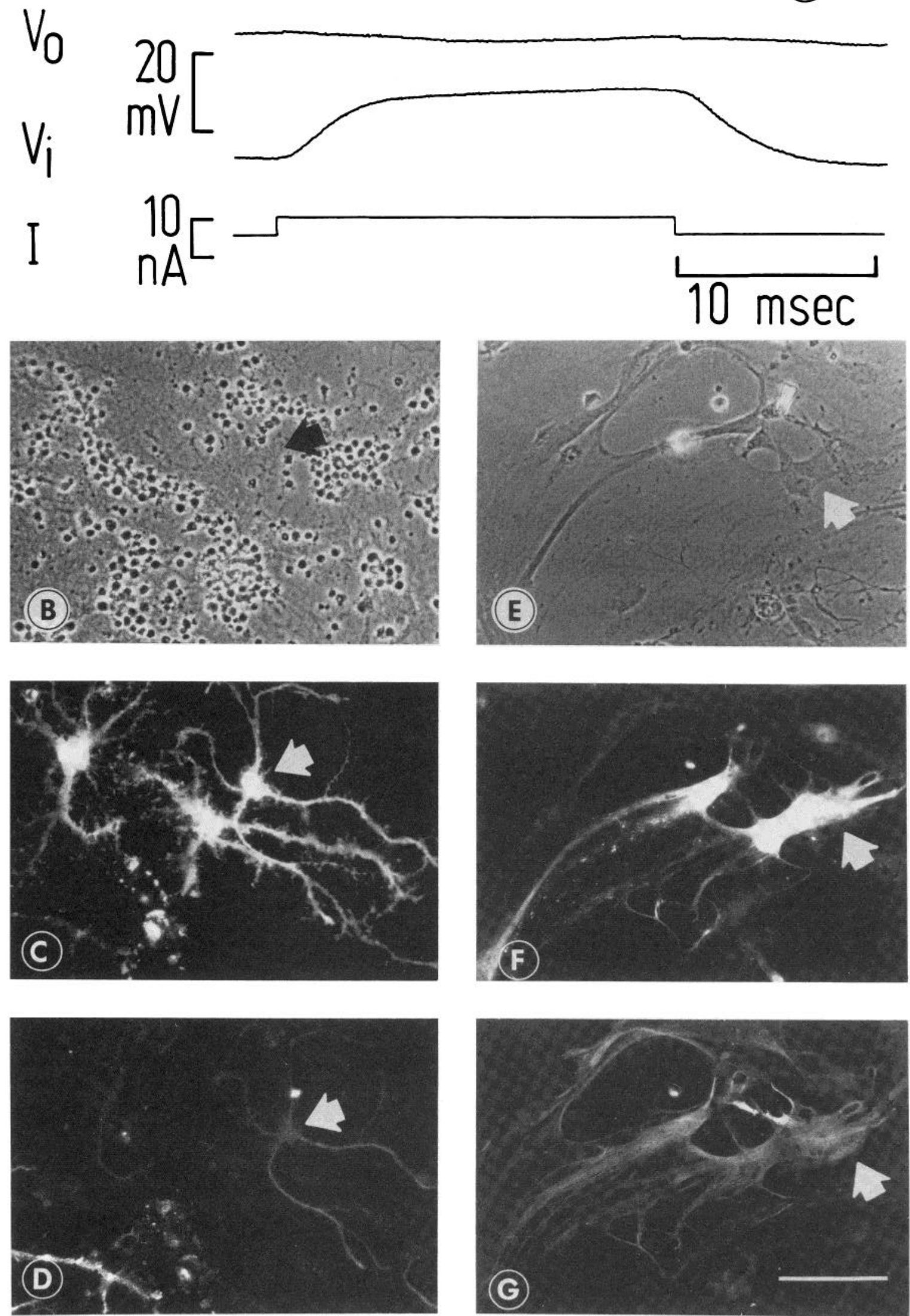

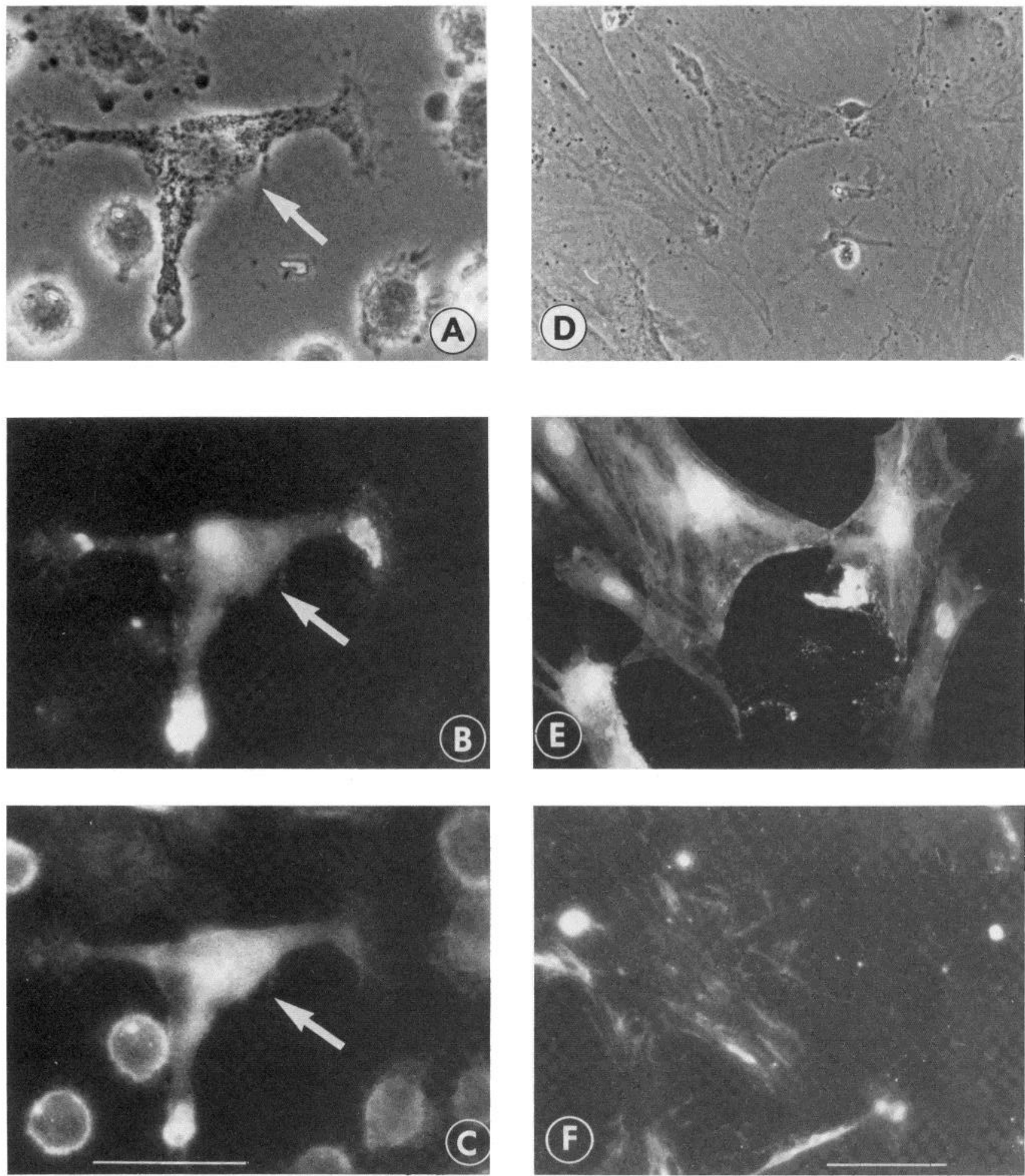

Figure 7. Macrophages and fibroblasts. $A$ to $C$ and $D$ to $F$ represent groups of identical microscopic fields. $A$ and $D$ are phase contrast micrographs of fluorescence images $B, C$ and $E, F$, respectively. $B$ and $E$ show the distribution of Lucifer Yellow and $C$ and $F$ the distribution of rhodamine immunolabeling. $A$ to $C$, an injected macrophage (arrows) identified by expression of $\mathrm{H}-2$ antigen shows no dye coupling to an adjacent cell which displays a particle with nonspecific fluorescence visible in $B$ and $C$. $D$ to $F$, Fibroblasts or fibroblast-like cells identified by an antibody to fibronectin readily permit the diffusion of dye from one cell to the other. Calibration bar represents $50 \mu \mathrm{m}$ for $A$ to $C$ and $100 \mu \mathrm{m}$ for $D$ to $F$.

presence of action potentials serves to identify neurons, we found it necessary to use immunological markers to distinguish the variety of non-neuronal elements. Morphological appearance is often helpful in making a pre- liminary identification. Once the eye has been guided by immunological criteria to recognize a certain cell type, it is possible, with practice, to select the desired cell type with good probability. Examples of the typical appear- 
ance of cultured cells are shown in Figures 2 to 7. Morphological criteria alone are, however, insufficient for conclusive recognition of cell types in culture, because cells may assume shapes which do not resemble those observed in situ and may also vary within the same culture dish.

Coupling in nervous systems has been studied in situ and in the culture system. Glial cells were investigated in invertebrates and were found to be coupled (Kuffler and Potter, 1964). Electrical and dye coupling has been demonstrated in amphibians (Kuffler et al., 1966; Orkand et al., 1973). In mammals Gutnick and colleagues (1981) showed dye coupling between physiologically identified glial cells and other unidentified cells presumed to be glia by their morphological appearance. In culture we are able to confirm the data of Moonen and Nelson (1978) for coupling in astrocytic cultures. While they penetrated cells in an approximately $90 \%$ enriched astrocytic culture system, we used glial fibrillary acidic protein for immunological identification of investigated cells. Coupling between identified oligodendrocytes was not previously shown. Coupling between Schwann cells could not be demonstrated under our culture conditions. We are unaware of studies on coupling among developing and adult Schwann cells. Dye coupling between crayfish axons and ad-axonal glia has been recently described (Viancour et al., 1981). Walker and Hild (1969) demonstrated coupling between glial cells and "quiet" neurons in culture. Since the cells were identified by morphological criteria only, it could well be that those "quiet" neurons were glial cells. This would explain the lack of excitability and the low input resistance of those cells. We never saw coupling between neurons and glial cells in cultures investigated in this study. Electrical coupling among neurons seems to be a property of restricted populations (Bennett, 1977; Gähwiler, 1981; Gutnick and Prince, 1981). In the culture system it has been demonstrated by Ransom and colleagues (1977b) in one of over 200 cases for spinal cord neurons. DRG neurons investigated in this study never showed any coupling to any other cell.

Low resistance pathways between glial cells presumably play a role in the redistribution of potassium ions which accumulate in the extracellular space during electrical activity of neurons. Potassium ions enter the glial cell in regions where the glial cells are depolarized, pass from cell to cell via gap junctions and leave the glial syncytium in regions where there is no potassium accumulation (Orkand et al., 1966; Gardner-Medwin et al., 1981). Although there is anatomical evidence for gap junctions between astrocytes and oligodendrocytes in normal central nervous system (Massa and Mugnaini, 1982), we were unable to demonstrate coupling between these cell types in our cultures. In the case where two populations of cells are strongly coupled to one another and weakly coupled to each other, the weak coupling could escape detection either with injection of current or dye. In our system the noise level of the recording system limited detection of coupling to coupling ratios of 0.005 and greater; the background tissue fluorescence placed an obvious, but undefined, limit on our ability to detect the intercellular spread of dye.

Dye coupling and electrical coupling are different approaches to measure the degree of intercellular commu- nications (Bennett, 1977; Bennett et al., 1981). As indications of intercellular connections both methods usually, but not always, yield the same results (Stewart, 1981) depending on morphology and the location of the intercellular gaps. In this study we found excellent agreement between the two methods in those cases where both were used. The dye injection method has the obvious advantage of requiring intracellular penetration of only one cell and yet it can reveal coupling among many cells. It is far more tedious and difficult to sample a whole range of cells with intracellular recording of current spread. In our study of oligodendrocyte coupling we found that two nearby oligodendrocytes may not be electrically coupled, but when one of the cells was dye injected, it could be seen to be coupled to other oligodendrocytes in the vicinity, showing that other factors in addition to the culture conditions play a role in the formation of gap junctions (Flagg-Newlon el al., 1979). One of those factors could be the degree of maturation of oligodendrocytes.

Michalke and Loewenstein (1971) looked for electrical coupling among a variety of mammalian cell types in culture, e.g., fibroblasts, liver, and lens cells, and found that coupling always occurred. They concluded that the ability to form communicating junctions was a basic and general property of mammalian cells in culture. More recently it has become apparent that there are restrictions to the formation of junctions in some heterologous culture systems (Loewenstein, 1979). In the adult tissues studied only skeletal muscle and nerve cells do not form heterologous intercellular junctions (Hyde et al., 1969). In this study we did not find indiscriminate coupling between closely apposed cells.

Specific intercellular connections are one of the distinguishing features of nervous systems. Such specific lines of communication appear to be maintained in nervous system cultures. This suggests that such systems will continue to be valuable for the study of the properties and development of cell interactions.

\section{References}

Bennett, M. V. L. (1977) Electrical transmission: A functional analysis and comparison to chemical transmission. In Cellular Biology of Neurons, E. R. Kandel, ed., pp. 357-416, Williams and Wilkins, Baltimore.

Bennett, M. V. L., D. C. Spray, and A. L. Harris (1981) Gap junctions and development. Trends Neurosci. 4: 159-163.

Bignami, A., L. F. Eng. D. Dahl, and C. T. Uyeda (1972) Localization of the glial fibrillary protein in astrocytes by immunofluorescence. Brain Res. 43: 429-435.

Eng, L. F., J. J. Vanderhaegen, A. Bignami, and B. Gerstl (1971) An acidic protein isolated from fibrous astrocytes. Brain Res. 28: $351-354$.

Fields, K. L., J. P. Brockes, R. Mirsky, and L. M. B. Wendon (1978) Cell surface markers for distinguishing different types of rat dorsal root ganglion cells in culture. Cell 14: 43-51.

Fischer, G. (1982) Cultivation of mouse cerebellar cells in serum free, hormonally defined media: Survival of neurons. Neurosci. Lett. 29: 297-303.

Flagg-Newton, J., I. Simpson, and W. R. Loewenstein (1979) Permeability of the cell to cell membrane channels in mammalian cell junctions. Science 205: 404-407.

Furshpan, E. J., and D. D. Potter (1968) Low-resistance junctions between cells in embryos and tissue culture. Curr. Topics Dev. Biol. 3: 95-127. 
Gähwiler, B. H. (1981) Labeling of neurons within CNS explants by intracellular injection of Lucifer Yellow. J. Neurobiol. 12: 187-191.

Gardner-Medwin, A. R., J. A. Coles, and M. Tsacopoulos (1981) Clearance of extracellular potassium: Evidence for spatial buffering by glial cells in the retina of the drone. Brain Res. 209: 452-457.

Gilula, N. B., and M. L. Epstein (1976) Cell-to-cell communication, gap junctions and calcium. 1975 Symp. Soc. Exp. Biol. 30: $257-272$.

Gutnick, M. J., and D. A. Prince (1981) Dye-coupling and possible electrical coupling in the guinea pig neocortical slice. Science 211: 67-70.

Gutnick, M. J., B. W. Connors, and B. R. Ransom (1981) Dyecoupling between glial cells in the guinea pig neocortical slice. Brain Res. 213: 486-492.

Hyde, A., B. Blondel, A. Matter, J. P. Cheneval, B. Filloux, and L. Girardier (1969) Homo- and heterocellular junctions in cell cultures: An electrophysiological and morphological study. Progr. Brain Res. 31: 283-311.

Kettenmann, H., U. Sonnhof, and M. Schachner (1983) Exclusive potassium dependence of the membrane potential in cultured mouse oligodendrocytes. J. Neurosci. 3: 500-505.

Kuffler, S. W., and D. D. Potter (1964) Glia in the leech central nervous system. Physiological properties and neuron-glia relationship. J. Neurophysiol. 27: 290-320.

Kuffler, S. W., J. G. Nicholls, and R. K. Orkand (1966) Physiological properties of glial cells in the central nervous system of amphibia. J. Neurophysiol. 29: 768-787.

Loewenstein, W. R. (1979) Junctional intercellular communication and the control of growth. Biochim. Biophys. Acta 560: 1-65.

Massa, P., and E. Mugnaini (1982) Cell junctions and intramembrane particles of astrocytes and oligodendrocytes: A freezefraction study. Neuroscience 7: 523-538.

Michalke, W., and W. R. Loewenstein (1971). Communication between cells of different type. Nature 232: 121-122.

Moonen, G., and P. G. Nelson (1978) Some physiological properties of astrocytes in primary cultures. In Dynamic Properties of Glial Cells, E. Schoffeniels, G. Frank, L. Hertz, and D. B. Tower, eds., Pergamon Press, London.

O'Lague, P., H. Dahlen, H. Rubin, and C. Tobias (1970) Electrical coupling: Low resistance junctions between mitotic and interphase fibroblasts in tissue culture. Science 170: 464-466.

Orkand, R. K., J. G. Nicholls, and S. W. Kuffler (1966) The effect of nerve impulses on the membrane potential of glial cells in the central nervous system of amphibia. J. Neuro- physiol. 29: 788-806.

Orkand, P. M., H. Bracho, and R. K. Orkand (1973) Glial metabolism: Alteration by potassium levels comparable to those during neural activity. Brain Res. 55: 467-471.

Ransom, B. R., E. Neale, M. Henkart, B. N. Bullock, and P. G. Nelson (1977a) Mouse spinal cord in cell culture. I. Morphology and intrinsic neuronal electrophysiologic properties. J. Neurophysiol. 40: 1132-1150.

Ransom, B. R., C. N. Christian, P. N. Bullock, and P. G. Nelson (1977b) Mouse spinal cord in cell culture. II. Synaptic activity circuit behaviour. J. Neurophysiol. 40: 1151-1162.

Schachner, M. (1982) Immunological analysis of cellular heterogeneity in the cerebellum. In Neuroimmunology, J. C. Brockes, ed., pp. 215-250, Plenum Press, New York.

Schachner, M., and M. Willinger (1979) Cell type specific cell surface antigens in the cerebellum. Progr. Brain Res. 51: 2344.

Schachner, M., T. Hedley-White, D. Hsu, G. Schoonmaker, and A. Bignami (1977) Ultrastructural localization of glial fibrillary acidic protein in mouse cerebellum by immunoperoxidase labeling. J. Cell Biol. 75: 67-73.

Schachner, M., G. Schoonmaker, and R. O. Hynes (1978) Cellular and subcellular localization of LETS protein in the nervous system. Brain Res. 158: 149-158.

Schachner, M., S. U. Kim, and R. Zehnle (1981) Developmental expression in central and peripheral nervous system of oligodendrocyte antigens ( $O$ antigens) recognized by monoclonal antibody. Dev. Biol. 83: 328-338.

Schnitzer, J., and M. Schachner (1981) Expression of Thy-1, H2 and NS-4 cell surface antigens and tetanus toxin receptors in early postnatal and adult mouse cerebellum. J. Neuroimmunol. 1: 429-456.

Sommer, I., and M. Schachner (1981) Monoclonal antibodies (01 to 04) to oligodendrocyte cell surfaces: An immunological study in the central nervous system. Dev. Biol. 83: 311-327.

Sonnhof, U., R. Foerderer, W. Schneider, and H. Kettenmann (1982) Cell puncturing with a step motor driven manipulator with simultaneous measurement of displacement. Pflüger's Arch. 392: 295-300.

Stewart, W. (1981) Lucifer dyes-Highly fluorescent dyes for biological tracing. Nature 292: 17-21.

Viancour, T. A., G. D. Billner, and M. L. Ballinger (1981) Selective transfer of Lucifer Yellow $\mathrm{CH}$ from axoplasm to adaxonal glia. Nature 293: 65-67.

Walker, F. D., and W. J. Hild (1969) Neuroglia electrically coupled to neurons. Science 165: 602-603. 